\title{
ON THE REGULARITY OF THE COMPLEX HESSIAN EQUATION
}

\author{
PER ÅHAG AND RAFA£ CZYŻ \\ We raise our cups to Urban Cegrell, gone but not forgotten, gone but ever here. \\ Until we meet again in Valhalla!
}

\begin{abstract}
This note aims to investigate the regularity of a solution to the Dirichlet problem for the complex Hessian equation, which has a density of the $m$-Hessian measure that belongs to $L^{q}$, for $q \leq \frac{n}{m}$.
\end{abstract}

\section{October 7, 2021}

\section{INTRODUCTION}

Let $n \geq 2,1 \leq m \leq n$. Consider the following Dirichlet problem for the complex Hessian equation in a bounded $m$-hyperconvex domain:

$$
\mathrm{H}_{m}\left(\mathrm{U}_{f}\right)=f d V_{2 n},
$$

or on a compact Kähler manifold:

$$
\mathrm{H}_{m}\left(\mathrm{U}_{f}\right)=f \omega^{n} .
$$

Here $d V_{2 n}$ is the Lebesgue measure in $\mathbb{R}^{2 n}, \omega$ is a Kähler form on $X$ such that $\int_{X} \omega^{n}=1$, and $f \in L^{\beta}$ is a density function. When $\beta>\frac{n}{m}$, Dinew and Kołodziej [14] proved that the solution $\mathrm{U}_{f}$ is a continuous $m$-subharmonic functions. Later on it was proved that the solution is in fact Hölder continuous (see e.g. 7, 10, 19, 24). Furthermore, if $\beta<\frac{n}{m}$, then the solution to (1.1) need not to be bounded (see 14]), which is a significant contrast to the case when $m=n$ (see e.g. [13, 18]).

Our aim is to consider the remaining singular cases, when $\beta \leq \frac{m}{n}$, to complete the overall picture. Under this assumption, we shall prove the regularity of a solution $u$ in the sense of being a member of Cegrell's energy class with bounded $(p, m)$ energy, $\mathcal{E}_{p, m}(\Omega)$. This is possible since both (1.1), and (1.2), are solvable within $\mathcal{E}_{p, m}(\Omega)$ (11, 21]). We say that a function is more regular if it belongs to $\mathcal{E}_{p, m}(\Omega)$ for a larger $p>0$, and this is motivated by the fact that $\mathcal{E}_{p, m}(\Omega) \subset \mathcal{E}_{q, m}(\Omega)$, for $q<p$. In this note, we shall use the following auxiliary notation: for $\beta>1$, we say that a $m$-subharmonic function $u$ belongs to the class $\mathcal{M}_{\beta}$, if $\mathrm{H}_{m}(u)=f d V_{2 n}$, and $f \in L^{\beta}(\Omega)$. Our regularity result for (1.1) is:

2020 Mathematics Subject Classification. Primary 32U05, 31C45,35B35; Secondary 32Q26, $53 \mathrm{C} 55,35 \mathrm{~J} 60$.

Key words and phrases. compact Kähler manifold, complex Hessian equation, $m$-subharmonic function $(\omega, m)$-subharmonic function, pluripotential theory, stability.

The second-named author was supported by the Priority Research Area SciMat under the program Excellence Initiative - Research University at the Jagiellonian University in Kraków. 
Theorem 3.4. Let $n \geq 2,1 \leq m<n$, and assume that $\Omega \subset \mathbb{C}^{n}$ is a $m$-hyperconvex domain. For $1<\beta \leq \frac{n}{m}$, let $u \in \mathcal{M}_{\beta}$. Then the following holds:

(1) if $\beta=\frac{n}{m}$, then $u \in \mathcal{E}_{p, m}(\Omega)$ for all $p>0$. Furthermore, $u \in L^{q}(\Omega)$, for all $q>0$

(2) if $\beta<\frac{n}{m}$, then $u \in \mathcal{E}_{p, m}(\Omega)$ for $p<\frac{(\beta-1) n m}{n-\beta m}$. Furthermore, $u \in L^{q}(\Omega)$, for all

$$
0<q<\frac{\beta n m}{n-\beta m}
$$

In Example 3.5. we provide an example that shows that the results in Theorem 3.4 are sharp. Furthermore, in Example 3.6 we show that in the case $\beta=\frac{n}{m}$, the solution can be unbounded. The corresponding result of Theorem 3.4 for the compact Kähler manifold case (1.2), is stated and proved in Theorem 5.3.

Commonly, stability results of equations of the type (1.1) is done by estimating the supremum norm of continuous, or bounded solutions, $\left|\mathrm{U}_{f}-\mathrm{U}_{f_{j}}\right|$, in terms of the $L^{q}$-norm of the difference of the densities, $\left\|f_{j}-f\right\|_{q}$. When it comes to the possibility of unbounded solutions, this type of estimation is not possible. Instead one can try to get weaker results, for examples $\mathrm{U}_{f_{j}}$ tends to $\mathrm{U}_{f}$ in capacity, or in $L^{q}$-norm, when $f_{j}$ converges to $f$. We shall approach it differently, and consider the convergence in the quasimetric space $\left(\mathcal{E}_{p, m}(\Omega), \mathrm{J}_{p}\right)$. Here the quasimetric $\mathrm{J}_{p}$ is defined by:

$$
\mathrm{J}_{p}(u, v)=\left(\int_{\Omega}|u-v|^{p}\left(\mathrm{H}_{m}(u)+\mathrm{H}_{m}(v)\right)\right)^{\frac{1}{p+m}}
$$

where $u, v \in \mathcal{E}_{p, m}(\Omega), p>0$. In [6], it was proved that $\left(\mathcal{E}_{p, m}(\Omega), \mathrm{J}_{p}\right)$ is a complete quasimetric space. Furthermore, convergence in $\left(X, \mathrm{~J}_{p}\right)$ implies convergence in capacity, and in $L^{q}$-norm, but the converse is not true. In this way, our approach is preferable. Our stability result states as follows.

Theorem 4.2. Let $n \geq 2,1 \leq m<n, 1<\beta \leq \frac{n}{m}$, and assume that $\Omega \subset \mathbb{C}^{n}$ is a $m$-hyperconvex domain. Furthermore, let $f_{j}, f \in L^{\beta}(\Omega)$ be such that $\left\|f_{j}-f\right\|_{\beta} \rightarrow 0$, as $j \rightarrow \infty$. Then for $p<p_{\infty}$ it holds that $\mathrm{J}_{p}\left(\mathrm{U}_{f_{j}}, \mathrm{U}_{f}\right) \rightarrow 0$. Here,

$$
p_{\infty}= \begin{cases}\frac{(\beta-1) n m}{n-\beta m}, & \text { if } \beta<\frac{n}{m} \\ \infty, & \text { if } \beta=\frac{n}{m} .\end{cases}
$$

Moreover, there exist a constant $C$ depending only on $\|f\|_{\beta}, \beta, m, p$, and a constant $\gamma$ depending only on $\beta, m, p$ such that

$$
\mathrm{J}_{p}\left(\mathrm{U}_{f_{j}}, \mathrm{U}_{f}\right) \leq C\left\|f_{j}-f\right\|_{\beta}^{\gamma} .
$$

The constant $\gamma$ can be taken as

$$
\begin{cases}\gamma=\frac{p}{m(p+m)} & , \text { when } p \leq \frac{m(\beta-1)}{\beta} ; \\ \gamma \in\left(0, \frac{\beta-1}{(p+m) \beta}\right)=\left(0, \frac{n-m}{n(m+p)}\right) & , \text { when } p>\frac{m(\beta-1)}{\beta}=\frac{m(n-m)}{n} \text { and } \beta=\frac{n}{m} \\ \gamma \in\left(0, \frac{\beta m(n+p)-n(m+p)}{m(p+m)(\beta(n+m)-n)}\right) & , \text { when } p>\frac{m(\beta-1)}{\beta} \text { and } \beta<\frac{n}{m} .\end{cases}
$$




\section{Preliminaries}

We start with the definition of $m$-subharmonic functions and the complex Hessian operator. Let $\Omega \subset \mathbb{C}^{n}, n \geq 2$, be a bounded domain, $1 \leq m \leq n$, and define $\mathbb{C}_{(1,1)}$ to be the set of $(1,1)$-forms with constant coefficients. Then, set

$$
\Gamma_{m}=\left\{\alpha \in \mathbb{C}_{(1,1)}: \alpha \wedge\left(d d^{c}|z|^{2}\right)^{n-1} \geq 0, \ldots, \alpha^{m} \wedge\left(d d^{c}|z|^{2}\right)^{n-m} \geq 0\right\} .
$$

Definition 2.1. Let $n \geq 2$, and $1 \leq m \leq n$. Assume that $\Omega \subset \mathbb{C}^{n}$ is a bounded domain, and let $u$ be a subharmonic function defined on $\Omega$. Then we say that $u$ is $m$-subharmonic if the following inequality holds

$$
d d^{c} u \wedge \alpha_{1} \wedge \cdots \wedge \alpha_{m-1} \wedge\left(d d^{c}|z|^{2}\right)^{n-m} \geq 0,
$$

in the sense of currents for all $\alpha_{1}, \ldots, \alpha_{m-1} \in \Gamma_{m}$. With $\mathcal{S H}_{m}(\Omega)$ we denote the set of all $m$-subharmonic functions defined on $\Omega$.

The real counterpart of $m$-subharmonic functions was first introduced by Caffarelli, Nirenberg, and Spruck [9]. The origin of these functions in the complex setting we focus on here was defined by Vinacua [27, 28. Later, Błocki [8] generalized this concept to the unbounded case we see in Definition 2.1, and pluripotential methods were introduced. For those who want more background on $m$-subharmonic functions, we refer to [1, 2, 15, 20].

Definition 2.2. Let $n \geq 2$, and $1 \leq m \leq n$. A bounded domain in $\Omega \subset \mathbb{C}^{n}$ is said to be $m$-hyperconvex if it admits a non-negative and $m$-subharmonic exhaustion function, i.e. there exits a $m$-subharmonic $\varphi: \Omega \rightarrow[0, \infty)$ such that the closure of the set $\{z \in \Omega: \varphi(z)<c\}$ is compact in $\Omega$, for every $c \in(-\infty, 0)$.

For further information on $m$-hyperconvex domains see [3]. Next, we shall recall the function classes that are of our interest. The following notations shall be used

$$
e_{0, m}(u)=\int_{\Omega} \mathrm{H}_{m}(u) \quad \text { and } \quad e_{p, m}(u)=\int_{\Omega}(-u)^{p} \mathrm{H}_{m}(u) .
$$

We say that a $m$-subharmonic function $\varphi$ defined on a $m$-hyperconvex domain $\Omega$ belongs to $\mathcal{E}_{m}^{0}(\Omega)$ if $\varphi$ is bounded,

$$
\lim _{z \rightarrow \xi} \varphi(z)=0 \quad \text { for every } \xi \in \partial \Omega
$$

and

$$
\int_{\Omega} \mathrm{H}_{m}(\varphi)<\infty
$$

Definition 2.3. Let $n \geq 2,1 \leq m \leq n$, and $p \geq 0$. Assume that $\Omega$ is a bounded $m$-hyperconvex domain in $\mathbb{C}^{n}$. We say that $u \in \mathcal{E}_{p, m}(\Omega)$ for $p \geq 0$, if $u$ is a $m$ subharmonic function defined on $\Omega$ such that there exists a decreasing sequence, $\left\{\varphi_{j}\right\}, \varphi_{j} \in \mathcal{E}_{m}^{0}(\Omega)$, that converges pointwise to $u$ on $\Omega$, as $j$ tends to $\infty$, and $\sup _{j} e_{p, m}\left(\varphi_{j}\right)<\infty$. Another common notation of the case $p=0, \mathcal{E}_{0, m}(\Omega)$, is $\mathcal{F}_{m}(\Omega)$.

In [20, 21], it was proved that for $u \in \mathcal{E}_{p, m}(\Omega)$ the complex Hessian operator, $\mathrm{H}_{m}(u)$, is well-defined, and

$$
\mathrm{H}_{m}(u)=\left(d d^{c} u\right)^{m} \wedge\left(d d^{c}|z|^{2}\right)^{n-m},
$$

where $d=\partial+\bar{\partial}$, and $d^{c}=\sqrt{-1}(\bar{\partial}-\partial)$. Theorem 2.4 is essential when working with $\mathcal{E}_{p, m}(\Omega), p>0$ (see Lu [20, 21], and Nguyen [25]). 
Theorem 2.4. Let $n \geq 2,1 \leq m \leq n$, and $p>0$. Assume that $\Omega$ is a bounded $m$-hyperconvex domain in $\mathbb{C}^{n}$. For $u_{0}, u_{1}, \ldots, u_{m} \in \mathcal{E}_{p, m}(\Omega)$, we have

$$
\begin{aligned}
\int_{\Omega}\left(-u_{0}\right)^{p} d d^{c} u_{1} \wedge \cdots \wedge d d^{c} u_{m} & \wedge\left(d d^{c}|z|^{2}\right)^{n-m} \\
\leq & C e_{p}\left(u_{0}\right)^{p /(p+m)} e_{p}\left(u_{1}\right)^{1 /(p+m)} \cdots e_{p}\left(u_{m}\right)^{1 /(p+m)},
\end{aligned}
$$

where $C \geq 1$ depends only on $p, m, n$ and $\Omega$.

\section{THE MAIN RESUlT}

In this section we shall prove the regularity of the solution, $\mathrm{U}_{f}$, to the following Dirichlet problem for the complex Hessian equation in a bounded $m$-hyperconvex domain $\Omega$,

$$
\mathrm{H}_{m}\left(\mathrm{U}_{f}\right)=f d V_{2 n} .
$$

It was proved by Dinew and Kołodziej [14, that if $\beta>\frac{n}{m}$, then $\mathrm{U}_{f}$ is continuous $m$-subharmonic functions, so $\mathrm{U}_{f} \in \mathcal{E}_{q, m}(\Omega)$, for all $q>0$. Therefore, we shall focus on the case $\beta \leq \frac{n}{m}$. Note that since $\mathrm{H}_{m}\left(\mathrm{U}_{f}\right)$ is bounded and if $\mathrm{U}_{f} \in \mathcal{E}_{p, m}(\Omega)$, then $\mathrm{U}_{f} \in \mathcal{E}_{q, m}(\Omega)$, for $q \leq p$.

Definition 3.1. Let $\beta>1$. We say that $m$-subharmonic function $u$ belongs to the class $\mathcal{M}_{\beta}$ if $\mathrm{H}_{m}(u)=f d V_{2 n}$ and $f \in L^{\beta}(\Omega)$.

Lemma 3.2. Let $\Omega$ be bounded m-hyperconvex domain. If $u \in \mathcal{E}_{p, m}(\Omega) \cap \mathcal{M}_{\beta}$, $p \geq 0$, then $u \in \mathcal{E}_{q, m}(\Omega)$, for

$$
\begin{cases}0<q<\frac{(\beta-1) n(m+p)}{\beta(n-m)}, & \text { when } \beta>\frac{n(m+p)}{m(n+p)} ; \\ 0<q \leq p, & \text { when } \beta \leq \frac{n(m+p)}{m(n+p)} .\end{cases}
$$

Moreover, there exists a constant $D>0$, which does not depend on $u$, such that

$$
e_{q, m}(u) \leq D\|f\|_{\beta}^{\frac{m+q}{m}} \text {. }
$$

Proof. Let $u_{j}$ be an approximating sequence defined by $\mathrm{H}_{m}\left(u_{j}\right)=\min (j, f) d V_{2 n}=$ $f_{j} d V_{2 n}, u_{j} \in \mathcal{E}_{m}^{0}(\Omega) \cap L^{\infty}(\Omega)$, and $u_{j} \searrow u$, as $j \rightarrow \infty$. First assume that $\beta>$ $\frac{n(m+p)}{m(n+p)}$, i.e. $\frac{(\beta-1) n(m+p)}{\beta(n-m)}>p$. For $\frac{1}{\alpha}+\frac{1}{\beta}=1$, we get

$$
\begin{aligned}
\int_{\Omega}\left(-u_{j}\right)^{q} \mathrm{H}_{m}\left(u_{j}\right)=\int_{\Omega}\left(-u_{j}\right)^{q} f_{j} & V_{2 n} \\
\leq & \left(\int_{\Omega}\left(-u_{j}\right)^{q \alpha} d V_{2 n}\right)^{\frac{1}{\alpha}}\left(\int_{\Omega} f_{j}^{\beta} d V_{2 n}\right)^{\frac{1}{\beta}},
\end{aligned}
$$

which is finite by the Sobolev type inequality (4, Theorem 5.4]) if $\alpha q<\frac{n(m+p)}{n-m}$. Hence,

$$
0<q<\frac{(\beta-1) n(m+p)}{\beta(n-m)} .
$$

By letting $j \rightarrow \infty$ we get desired result. Again, by the Sobolev type inequality (4 Theorem 5.4]) we have

$$
\|u\|_{\alpha q} \leq C e_{q, m}(u)^{\frac{1}{m+q}},
$$

where the constant $C$ does not depend on $u$. Therefore, (3.1) yields

$$
e_{q}(u) \leq C^{q} e_{q}(u)^{\frac{q}{m+q}}\|f\|_{\beta},
$$


and we arrive at

$$
e_{q, m}(u) \leq D\|f\|_{\beta}^{\frac{m+q}{q}} .
$$

Now assume that $\beta \leq \frac{n(m+p)}{m(n+p)}$, i.e. $\frac{(\beta-1) n(m+p)}{\beta(n-m)} \leq p$. By repeating the above argument we get $u \in \mathcal{E}_{q, m}(\Omega)$ for $0<q \leq \frac{(\beta-1) n(m+p)}{\beta(n-m)}$, and for $p=q$. Now we can use a standard argument to show that actually $u \in \mathcal{E}_{q, m}(\Omega)$ when $0<q \leq p$.

By Lemma 3.2 we see that the only interested case for us is when $\beta \in\left(\frac{n(m+p)}{m(n+p)}, \frac{n}{m}\right]$. Under this assumption we will in the next lemma improve Lemma 3.2 further.

Lemma 3.3. Let $\Omega$ be bounded m-hyperconvex domain, and assume that $\beta \in$ $\left(\frac{n(m+p)}{m(n+p)}, \frac{n}{m}\right]$. If $u \in \mathcal{E}_{p, m}(\Omega) \cap \mathcal{M}_{\beta}$, then $u \in \mathcal{E}_{q, m}(\Omega)$, for

$$
\begin{cases}0<q<\frac{(\beta-1) n m}{n-\beta m}, & \text { when } \beta<\frac{n}{m} \\ q>0, & \text { when } \beta=\frac{n}{m}\end{cases}
$$

Proof. We base this proof on the following iterating procedure: When $\beta \in\left(\frac{n(m+p)}{m(n+p)}, \frac{n}{m}\right]$, then if $u \in \mathcal{E}_{p, m}(\Omega)$, then $u \in \mathcal{E}_{q, m}(\Omega)$, for $q<\frac{(\beta-1) n(m+p)}{\beta(n-m)}=p^{\prime}(>p)$. Moreover, if $\beta>\frac{n(m+p)}{m(n+p)}$, then $\beta>\frac{n\left(m+p^{\prime}\right)}{m\left(n+p^{\prime}\right)}$, and we can continue repeating the procedure. Again, if $u \in \mathcal{E}_{p^{\prime}, m}(\Omega)$, then $u \in \mathcal{E}_{q^{\prime}, m}(\Omega)$, for $q^{\prime}<\frac{(\beta-1) n\left(m+p^{\prime}\right)}{\beta(n-m)}$. In this way, in each step, the new $q^{\prime}$ is slightly bigger than $p^{\prime}$. Thus, we obtain the following sequence

$$
p_{N}=\alpha\left(m+p_{N-1}\right), \text { where } \alpha=\frac{(\beta-1) n}{\beta(n-m)} .
$$

If $\alpha=1$, then $\beta=\frac{n}{m}$ and $p_{N}=m+p_{N-1}$. Hence, $p_{N} \rightarrow \infty$, so $u \in \mathcal{E}_{p, m}(\Omega)$ for all $p>0$. Now assume that $\alpha<1$. We have that the above sequence is convergent, since it is increasing and bounded from above by $p_{\infty}=\frac{(\beta-1) n m}{n-\beta m}$. Therefore, we get $p_{N} \rightarrow p_{\infty}=\frac{(\beta-1) n m}{n-\beta m}$, as $N \rightarrow \infty$. Hence, $u \in \mathcal{E}_{q, m}(\Omega)$ for $q<p_{\infty}$.

Remark. Note that the exponent $q$ obtained in Lemma 3.3 is better than the exponent obtained in Lemma 3.2, since

$$
\text { if } \beta>\frac{n(m+p)}{m(n+p)} \text {, then } \frac{(\beta-1) n(m+p)}{\beta(n-m)}<\frac{(\beta-1) n m}{n-\beta m} \text {. }
$$

Theorem 3.4. Let $n \geq 2,1 \leq m<n$, and assume that $\Omega \subset \mathbb{C}^{n}$ is a $m$-hyperconvex domain. For $1<\beta \leq \frac{n}{m}$, let $u \in \mathcal{M}_{\beta}$. Then the following holds:

(1) if $\beta=\frac{n}{m}$, then $u \in \mathcal{E}_{p, m}(\Omega)$ for all $p>0$. Furthermore, $u \in L^{q}(\Omega)$, for all $q>0$;

(2) if $\beta<\frac{n}{m}$, then $u \in \mathcal{E}_{p, m}(\Omega)$ for $p<\frac{(\beta-1) n m}{n-\beta m}$. Furthermore, $u \in L^{q}(\Omega)$, for all

$$
0<q<\frac{\beta n m}{n-\beta m} .
$$

Proof. Let $u \in \mathcal{M}_{\beta}$, then $u \in \mathcal{E}_{0, m}$, since $\mathrm{H}_{m}(u)$ is bounded. Now we can use Lemma 3.2 to get $u \in \mathcal{E}_{p, m}(\Omega)$, for $p<\frac{(\beta-1) n m}{\beta(n-m)}=p_{0}$. Observe that $\beta>\frac{n\left(m+p_{0}\right)}{m\left(n+p_{0}\right)}$. 
Now by Lemma 3.3 we get the thesis. The integrability condition for $u$ follows from [4].

In Example 3.5 we provide an example that shows that our result is sharp, and in Example 3.6 we show that in the case $\beta=\frac{n}{m}$, the solution can be unbounded.

Example 3.5. Let $u_{\alpha}(z)=1-|z|^{\alpha}$, for $0>\alpha>2-\frac{2 n}{m}$, be a function defined in the unit ball $B(0,1) \subset \mathbb{C}^{n}, n \geq 2$. Then $u_{\alpha}$ is $m$-subharmonic, and $\mathrm{H}_{m}\left(u_{\alpha}\right)=$ $c f_{\alpha}(z) d V_{2 n}$. Here, $c$ is a constant, and the density is given by $f_{\alpha}(z)=|z|^{(\alpha-2) m}$. For $\beta \in\left(1, \frac{n}{m}\right)$, the function $f_{\alpha}$ is in $L^{\beta}(B(0,1))$ if, and only if, $\alpha>2-\frac{2 n}{m \beta}$. Finally, $u_{\alpha} \in \mathcal{E}_{p, m}(B(0,1))$ if, and only if, $p<\frac{-2 n+(2-\alpha) m}{\alpha}$. Then, choose $\epsilon>0$ small enough, and let $\alpha(\epsilon)=2-\frac{2 n}{m \beta}+\frac{2 \epsilon}{m \beta}$. Then $u_{\alpha(\epsilon)} \notin \mathcal{E}_{p(\epsilon), m}(B(0,1))$ for

$$
p(\epsilon)=\frac{m n(\beta-1)+m \epsilon}{n-m \beta-\epsilon} \text {. }
$$

Example 3.6. Let $0<\alpha<\frac{n-1}{n}$, and define $u_{\alpha}(z)=(\ln 2)^{\alpha}-(-\ln |z|)^{\alpha}$. Then $u_{\alpha}$ is a unbounded $m$-subharmonic function defined in the ball $B\left(0, \frac{1}{2}\right) \subset \mathbb{C}^{n}, n \geq 2$. We have,

$$
\mathrm{H}_{m}\left(u_{\alpha}\right)=c f_{\alpha}(z) d V_{2 n},
$$

where $c$ is a constant, and the density is given by

$$
f_{\alpha}(z)=|z|^{-2 m}(-\ln |z|)^{\alpha m-m-1}((2 n-2 m)(-\ln |z|)+m(1-\alpha)) .
$$

Finally, note that $f_{\alpha} \in L^{\frac{n}{m}}\left(B\left(0, \frac{1}{2}\right)\right)$, since

$$
\int_{B\left(0, \frac{1}{2}\right)}\left(f_{\alpha}\right)^{\frac{n}{m}} d V_{2 n} \leq C \int_{0}^{\frac{1}{2}} \frac{d t}{t(-\ln t)^{n(1-\alpha)}}<\infty .
$$

\section{Stability}

Before proving our stability result, Theorem 4.2, let us recall some necessary tools and results. For $u, v \in \mathcal{E}_{p, m}(\Omega), p>0$, define

$$
\mathrm{J}_{p}(u, v)=\left(\int_{\Omega}|u-v|^{p}\left(\mathrm{H}_{m}(u)+\mathrm{H}_{m}(v)\right)\right)^{\frac{1}{p+m}}
$$

then it follows from [6] that $\left(\mathcal{E}_{p, m}(\Omega), \mathrm{J}_{p}\right)$ is a complete quasimetric space. We shall as well need the following comparison principle from [26].

Theorem 4.1. Let $\Omega$ be a bounded m-hyperconvex domain, let $u, v \in \mathcal{E}_{0, m}(\Omega)(p=$ $0), w \in \mathcal{E}_{m}^{0}(\Omega)$, then

$$
\int_{\{u<v\}}(v-u)^{m} \mathrm{H}_{m}(w) \leq m !\|w\|_{\infty}^{m-1} \int_{\{u<v\}}(-w)\left(\mathrm{H}_{m}(u)-\mathrm{H}_{m}(v)\right) .
$$

Theorem 4.2. Let $n \geq 2,1 \leq m<n, 1<\beta \leq \frac{n}{m}$, and assume that $\Omega \subset \mathbb{C}^{n}$ is a m-hyperconvex domain. Furthermore, let that $f_{j}, f \in L^{\beta}(\Omega)$ be such that $\left\|f_{j}-f\right\|_{\beta} \rightarrow 0$, as $j \rightarrow \infty$. Then for $p<p_{\infty}$ it holds that $\mathrm{J}_{p}\left(\mathrm{U}_{f_{j}}, \mathrm{U}_{f}\right) \rightarrow 0$. Here,

$$
p_{\infty}= \begin{cases}\frac{(\beta-1) n m}{n-\beta m}, & \text { if } \beta<\frac{n}{m} \\ \infty, & \text { if } \beta=\frac{n}{m} .\end{cases}
$$


Moreover, there exist a constant $C$ depending only on $\|f\|_{\beta}, \beta, m, p$, and a constant $\gamma$ depending only on $\beta, m, p$ such that

$$
\mathrm{J}_{p}\left(\mathrm{U}_{f_{j}}, \mathrm{U}_{f}\right) \leq C\left\|f_{j}-f\right\|_{\beta}^{\gamma} .
$$

The constant $\gamma$ can be taken as

$$
\begin{cases}\gamma=\frac{p}{m(p+m)} & , \text { when } p \leq \frac{m(\beta-1)}{\beta} \\ \gamma \in\left(0, \frac{\beta-1}{(p+m) \beta}\right)=\left(0, \frac{n-m}{n(m+p)}\right) & , \text { when } p>\frac{m(\beta-1)}{\beta}=\frac{m(n-m)}{n} \text { and } \beta=\frac{n}{m} \\ \gamma \in\left(0, \frac{\beta m(n+p)-n(m+p)}{m(p+m)(\beta(n+m)-n)}\right) & , \text { when } p>\frac{m(\beta-1)}{\beta} \text { and } \beta<\frac{n}{m}\end{cases}
$$

Proof. Let $\frac{1}{\alpha}+\frac{1}{\beta}=1$, and let $p_{0}=\frac{m(\beta-1)}{\beta}$. Then, choose $\varphi_{0} \in \mathcal{E}_{m}^{0}(\Omega)$ such that $\mathrm{H}_{m}\left(\varphi_{0}\right)=d V_{2 n}$. Since $\left\|f_{j}-f\right\|_{\beta} \rightarrow 0$, as $j \rightarrow \infty$, we can assume

$$
\left\|f_{j}-f\right\|_{\beta} \leq \frac{1}{2^{j}}
$$

Thanks to Theorem 4.1,

$$
\begin{gathered}
\mathrm{J}_{p_{0}}\left(\mathrm{U}_{f_{j}}, \mathrm{U}_{f}\right)^{p_{0}+m}=\int_{\Omega}\left|\mathrm{U}_{f_{j}}-\mathrm{U}_{f}\right|^{p_{0}}\left(\mathrm{H}_{m}\left(\mathrm{U}_{f_{j}}\right)+\mathrm{H}_{m}\left(\mathrm{U}_{f}\right)\right) \\
=\int_{\Omega}\left|\mathrm{U}_{f_{j}}-\mathrm{U}_{f}\right|^{p_{0}}\left(f_{j}+f\right) d V_{2 n} \leq\left(\int_{\Omega}\left|\mathrm{U}_{f_{j}}-\mathrm{U}_{f}\right|^{p_{0} \alpha} d V_{2 n}\right)^{\frac{1}{\alpha}}\left\|f_{j}+f\right\|_{\beta} \\
\leq\left(m !\left\|\varphi_{0}\right\|_{\infty}^{m} \int_{\Omega}\left|f_{j}-f\right| d V_{2 n}\right)^{\frac{1}{\alpha}}\left\|f_{j}+f\right\|_{\beta} \\
\leq\left(m !\left\|\varphi_{0}\right\|_{\infty}^{m}\right)^{\frac{\beta-1}{\beta}}\left(2\|f\|_{\beta}+1\right) V_{2 n}(\Omega)^{\frac{(\beta-1)^{2}}{\beta^{2}}}\left\|f_{j}-f\right\|_{\beta}^{\frac{\beta-1}{\beta}}=C\left\|f_{j}-f\right\|_{\beta}^{\frac{p_{0}}{m}} .
\end{gathered}
$$

If $p<\frac{m(\beta-1)}{\beta}=p_{0}$, then

$$
\begin{aligned}
\mathrm{J}_{p}\left(\mathrm{U}_{f_{j}}, \mathrm{U}_{f}\right)^{p+m}=\int_{\Omega}\left|\mathrm{U}_{f_{j}}-\mathrm{U}_{f}\right|^{p}\left(\mathrm{H}_{m}\left(\mathrm{U}_{f_{j}}\right)+\mathrm{H}_{m}\left(\mathrm{U}_{f}\right)\right) & \\
& \leq \mathrm{J}_{p_{0}}\left(\mathrm{U}_{f_{j}}, \mathrm{U}_{f}\right)^{\frac{p\left(p_{0}+m\right)}{p_{0}}}\left\|f_{j}+f\right\|_{1}^{\frac{p_{0}-p}{p_{0}}},
\end{aligned}
$$

and we can use the estimation above to obtain

$$
\mathrm{J}_{p}\left(\mathrm{U}_{f_{j}}, \mathrm{U}_{f}\right) \leq C\left\|f_{j}-f\right\|_{\beta}^{\frac{p}{m(p+m)}} .
$$

Next, assume that $p_{\infty}>p>p_{0}$, and choose $p^{\prime} \in\left(p, p_{\infty}\right)$. Therefore, from $\mathrm{U}_{f_{j}}, \mathrm{U}_{f} \in \mathcal{E}_{p^{\prime}, m}(\Omega)$ it follows

$$
\begin{aligned}
& \mathrm{J}_{p}\left(\mathrm{U}_{f_{j}}, \mathrm{U}_{f}\right)^{p+m}=\int_{\Omega}\left|\mathrm{U}_{f_{j}}-\mathrm{U}_{f}\right|^{p}\left(\mathrm{H}_{m}\left(\mathrm{U}_{f_{j}}\right)+\mathrm{H}_{m}\left(\mathrm{U}_{f}\right)\right) \\
& \quad \leq\left(\mathrm{J}_{p_{0}}\left(\mathrm{U}_{f_{j}}, \mathrm{U}_{f}\right)\right)^{\frac{\left(m+p_{0}\right)\left(p^{\prime}-p\right)}{p^{\prime}-p_{0}}}\left(\int_{\Omega}\left|\mathrm{U}_{f_{j}}-\mathrm{U}_{f}\right|^{p^{\prime}}\left(\mathrm{H}_{m}\left(\mathrm{U}_{f_{j}}\right)+\mathrm{H}_{m}\left(\mathrm{U}_{f}\right)\right)\right)^{\frac{p-p_{0}}{p^{\prime}-p_{0}}} .
\end{aligned}
$$


From Theorem 2.4, and Lemma 3.2, we get that the second term in (4.1) is bounded by

$$
\begin{aligned}
\int_{\Omega}\left|\mathrm{U}_{f_{j}}-\mathrm{U}_{f}\right|^{p^{\prime}}\left(\mathrm{H}_{m}\left(\mathrm{U}_{f_{j}}\right)+\mathrm{H}_{m}\left(\mathrm{U}_{f}\right)\right) \leq e_{p^{\prime}}\left(\mathrm{U}_{f_{j}}+\mathrm{U}_{f}\right) & \\
\leq D^{\frac{m+p^{\prime}}{m}}\left(e_{p^{\prime}}\left(\mathrm{U}_{f_{j}}\right)^{\frac{1}{m+p^{\prime}}}+e_{p^{\prime}}\left(\mathrm{U}_{f}\right)^{\frac{1}{m+p^{\prime}}}\right)^{m+p^{\prime}} & \leq D^{\prime}\left(\left\|f_{j}\right\|_{\beta}^{\frac{1}{m}}+\|f\|_{\beta}^{\frac{1}{m}}\right)^{m+p^{\prime}} \\
& \leq D^{\prime}\left(\left(\|f\|_{\beta}+1\right)^{\frac{1}{m}}+\|f\|_{\beta}^{\frac{1}{m}}\right)^{m+p^{\prime}}
\end{aligned}
$$

where the constants $D$ and $D^{\prime}$ do not depend on $\mathrm{U}_{f_{j}}$ and $\mathrm{U}_{f}$. Finally, we obtain

$$
\begin{aligned}
& \mathrm{J}_{p}\left(\mathrm{U}_{f_{j}}, \mathrm{U}_{f}\right) \leq C\left\|f_{j}-f\right\|_{\beta}^{\frac{p_{0}\left(p^{\prime}-p\right)}{m(p+m)\left(p^{\prime}-p_{0}\right)}}=C\left\|f_{j}-f\right\|_{\beta}^{\frac{(\beta-1)\left(p^{\prime}-p\right)}{(p+m)\left(\beta p^{\prime}-m(\beta-1)\right)}} \\
&=C\left\|f_{j}-f\right\|_{\beta}^{\gamma} .
\end{aligned}
$$

Passing to the limit with $p^{\prime} \rightarrow p_{\infty}$, we get desired conclusion.

\section{KÄHLER MANIFOLD CASE}

Let $n \geq 2, p \geq 0$, and let $1 \leq m<n$. Assume that $(X, \omega)$ is a connected and compact Kähler manifold of complex dimension $n$, where $\omega$ is a Kähler form on $X$ such that $\int_{X} \omega^{n}=1$. For any $u \in \mathcal{S H}_{m}(X, \omega)$, let

$$
\omega_{u}=d d^{c} u+\omega .
$$

and the complex Hessian operator is defined by

$$
\mathrm{H}_{m}(u):=\omega_{u}^{m} \wedge \omega^{n-m} .
$$

We define the class of $(\omega, m)$-subharmonic functions with bounded $(p, m)$-energy as

$$
\mathcal{E}_{p, m}(X, \omega):=\left\{u \in \mathcal{E}_{m}(X, \omega): u \leq 0, \int_{X}(-u)^{p} \mathrm{H}_{m}(u)<\infty\right\},
$$

where

$$
\mathcal{E}_{m}(X, \omega)=\left\{u \in \mathcal{S H}_{m}(X, \omega): \int_{X} \mathrm{H}_{m}(u)=1\right\} .
$$

Additionally, information on $(\omega, m)$-subharmonic functions defined on compact Kähler manifolds we refer to [5, 16, 17, 22, 23].

In this section, we shall arrive in Theorem 5.3 to a regularity result on compact Kähler manifold corresponding to that of Theorem 3.4. With a few minor changes we are able to follow Section 3 . The counterpart of Lemma 3.2 is as follows.

Lemma 5.1. If $u \in \mathcal{E}_{p, m}(X, \omega) \cap \mathcal{M}_{\beta}, p \geq 0$, then $u \in \mathcal{E}_{q, m}(X, \omega)$, for

$$
\begin{cases}0<q<\frac{(\beta-1) n(1+p)}{\beta(n-m)}, & \text { when } \beta>\frac{n(1+p)}{n+m p} ; \\ 0<q \leq p, & \text { when } \beta \leq \frac{n(1+p)}{n+m p} .\end{cases}
$$

From [5. Theorem 4.4], it follows that if $u \in \mathcal{E}_{p, m}(X, \omega)$, then $u \in L^{q}(X)$, for $q<\frac{(p+1) n}{n-m}$, then again using the reasoning from Section 3 we get the counterpart of Lemma 3.3 . 
Lemma 5.2. If $u \in \mathcal{E}_{p, m}(X, \omega) \cap \mathcal{M}_{\beta}$, then $u \in \mathcal{E}_{q, m}(X, \omega)$, for

$$
\begin{cases}0<q<\frac{n(\beta-1)}{n-\beta m}, & \text { when } \frac{n(1+p)}{n+m p}<\beta<\frac{n}{m} ; \\ q>0, & \text { when } \beta=\frac{n}{m} .\end{cases}
$$

Since, if $u \in \mathcal{S H}_{m}(X, \omega)$, then $u \in L^{q}(X)$, for $q<\frac{n}{n-m}$ ([22, Corollary 6.7]), we get the analogue of Theorem 3.4 .

Theorem 5.3. For $1<\beta \leq \frac{n}{m}$, let $u \in \mathcal{M}_{\beta} \cap \mathcal{E}_{m}(X, \omega)$. Then the following holds:

(1) if $\beta=\frac{n}{m}$, then $u \in \mathcal{E}_{p, m}(X, \omega)$, for all $p>0$. Furthermore, $u \in L^{q}(X)$, for all $q>0$;

(2) if $\beta<\frac{n}{m}$, then $u \in \mathcal{E}_{p, m}(X, \omega)$, for $p<\frac{n(\beta-1)}{n-\beta m}=p_{0}$. Furthermore, $u \in$ $L^{q}(X)$, for all

$$
q<\frac{\left(p_{0}+1\right) n}{n-m}=\frac{\beta n}{n-\beta m} .
$$

\section{REFERENCES}

[1] Abdullaev B. I., Sadullaev A., Potential theory in the class of $m$-subharmonic functions. Proc. Steklov Inst. Math. 279 (2012), no. 1, 155-180.

[2] Abdullaev B. I., Sadullaev A., Capacities and Hessians in the class of $m$-subharmonic functions. Dokl. Math. 87 (2013), no. 1, 88-90.

[3] Åhag P., Czyż R., Hed L., The geometry of $m$-hyperconvex domains. J. Geom. Anal. 28 (2018), no. 4, 3196-3222.

[4] Åhag P., Czyż R., Poincaré- and Sobolev-type inequalities for complex $m$-Hessian equations. Results Math. 75 (2020), no. 2, Paper No. 63, 21 pp.

[5] Åhag P., Czyż R., A characterization of the degenerate complex Hessian equations for functions with bounded $(p, m)$-energy. Manuscript (2020), arXiv:2003.06157

[6] Åhag P., Czyż R., On a family of quasimetric spaces in generalized potential theory. Manuscript (2021), arXiv: .

[7] Bouhssina M., On the regularity of complex Hessian equation on $m$-hyperconvex domain. Complex Var. Elliptic Equ. 64 (2019), no. 10, 1739-1755.

[8] Błocki Z., Weak solutions to the complex Hessian equation. Ann. Inst. Fourier (Grenoble) 55 (2005), no. 5, 1735-1756.

[9] Caffarelli L., Nirenberg L., Spruck J., The Dirichlet problem for nonlinear second-order elliptic equations. III. Functions of the eigenvalues of the Hessian. Acta Math. 155 (1985), no. 3-4, 261-301.

[10] Charabati M., Modulus of continuity of solutions to complex Hessian equations. Internat. J. Math. 27 (2016), no. 1, 1650003, 24 pp.

[11] Cegrell U., Pluricomplex energy. Acta Math. 180 (1998), no. 2, 187-217.

[12] Cegrell U., Kołodziej S., The equation of complex Monge-Ampère type and stability of solutions. Math. Ann. 334 (2006), no. 4, 713-729.

[13] Cegrell U., Persson L., The Dirichlet problem for the complex Monge-Ampère operator: stability in $L^{2}$. Michigan Math. J. 39 (1992), no. 1, 145-151.

[14] Dinew S., Kołodziej S, A priori estimates for complex Hessian equations. Anal. PDE 7 (2014), no. $1,227-244$.

[15] Dinew S., Kołodziej S, Non standard properties of $m$-subharmonic functions. Dolomites Res. Notes Approx. 11 (2018), Special Issue Norm Levenberg, 35-50.

[16] Dinew S. Lu H.-C., Mixed Hessian inequalities and uniqueness in the class $\mathcal{E}(X, \omega, m)$. Math. Z. 279 (2015), no. 3-4, 753-766.

[17] Guedj V., Lu H.-C., Zeriahi A., Plurisubharmonic envelopes and supersolutions. J. Differential Geom. 113 (2019), no. 2, 273-313.

[18] Kołodziej S., The complex Monge-Ampère equation. Acta Math. 180 (1998), no. 1, 69-117.

[19] Kołodziej, Nguyen N.C., An inequality between complex Hessian measures of Hölder continuous $m$-subharmonic functions and capacity. Geometric analysis. In honor of Gang Tian's 60th birthday, pp. 157-166, Progress in Mathematics 333. Birkhäuser (2020). 
[20] Lu H.-C., Complex Hessian equations. Doctoral thesis. University of Toulouse III Paul Sabatier, 2012.

[21] Lu H.-C., A variational approach to complex Hessian equations in $\mathbb{C}^{n}$. J. Math. Anal. Appl. 431 (2015), no. 1, 228-259.

[22] Lu H.-C., Nguyen V.-D., Degenerate complex Hessian equations on compact Kähler manifolds. Indiana Univ. Math. J. 64 (2015), no. 6, 1721-1745.

[23] Lu H.-C., Nguyen V.-D., Complex Hessian equations with prescribed singularity on compact Kähler manifolds. Ann. Sc. Norm. Super. Pisa Cl. Sci. (accepted). Manuscript (2019), arXiv:1909.02469

[24] Nguyen N.C., Hölder continuous solutions to complex Hessian equations. Potential Anal. 41 (2014), no. 3, 887-902.

[25] Nguyen V.T. , On delta $m$-subharmonic functions. Ann. Polon. Math. 118 (2016), no. 1, $25-49$.

[26] Nguyen V.T., Maximal $m$-subharmonic functions and the Cegrell class $\mathcal{N}_{m}$. Indag. Math. (N.S.) 30 (2019), no. 4, 717-739.

[27] Vinacua A., Nonlinear elliptic equations written in terms of functions of the eigenvalues of the complex Hessian. Doctoral thesis, New York University, 1986.

[28] Vinacua A., Nonlinear elliptic equations and the complex Hessian. Comm. Partial Differential Equations 13 (1988), no. 12, 1467-1497.

Department of Mathematics and Mathematical Statistics, Umeå University, SE-901 87 UMEÅ, SWEDEN

Email address: per.ahag@umu.se

Institute of Mathematics, Faculty of Mathematics and Computer Science, JagielLONian University, Łojasiewicza 6, 30-348 Kraków, Poland

Email address: Rafal.Czyz@im.uj.edu.pl 